\title{
REFLECTIONS ABOUT THE METHODOLOGICAL DUALISM IN HEALTH RESEARCH
}

Antonia Regina Ferreira Furegato ${ }^{1}$

Nursing assembles knowledge from different theoretical sciences and applied areas, involving the biological, human and social. Production of this specific knowledge aims to contribute to improve the quality of care and obtain better levels of health for human beings.

Assuming truth of knowledge is the basis that determines the understanding of how people relate to each other. It is worth questioning the adjectives connected with it and actions taken towards it, in contexts that can be real or not: what is the range of rationality of knowledge? What is the extent of the diversity of knowledge? Is it possible to trust this knowledge and its rationality?

Overcoming antagonisms among perspectives previously considered opposite, excluding and incompatible, in their epistemological and ontological positions towards the nature of research and society, makes it obsolete to defend the descriptive positivism of numbers in contrast to the descriptive qualitative production of words.

Nevertheless, forms of knowledge legitimacy need to be reconsidered, approaching factual truth (quantitative) to the truth of discourse (qualitative).

Quantitative, positivist research aims to document objective facts of reality and is interested in the numerical expression of phenomena. This presumes the separation between observing subject and observed "object", and supports the existence of an objective reality, subject to "immutable" laws.

Qualitative research aims to reconstruct symbolical patterns, expressed in an intersubjective way; is interested in heterogeneity and relational logic, expressed in words and images. It accepts the role of ideology as well as subjectivity.

In principle, qualitative and quantitative research are distinct paradigmatic forms of conceiving reality, of conceiving the determining axes of social tissue, methodological instruments and strategies, and ideological reflections about human problems.

Nonetheless, exact sciences are not free of values since, in the act of knowing, there is interest in transforming. On the other hand, subjectivity alone does not manage to cover the context it is formed in or which it transforms. Besides that, the intangible nature of quality "falsely" suggests its imponderability.

The mathematization of the world coincides with the appearance of the bourgeois society. The 20th century marks the strengthening of the theoretical and epistemological inquisitive bases, from the quantitative and statistical perspective. It tries to find laws independently of the social, cultural, historical and political contexts. The cause-effect relation is present in the design of dependent and independent variables, where knowledge of the phenomena is valid if it is countable, measurable or provable.

Marxism caused an intense reflection about the basis of knowledge, but also an overvaluation of the social.

Neo-positivism excludes objective naiveté, recognizes the lack of radical subject/object maintenance and uses different methods in a complementary way. It considers science free from preconceived values, searching for explanation, prediction and control of phenomena, not only in numbers but also in words.

The constructivist paradigm is opposed to positivism as it does not intend to predict, control or transform the natural world, but tries to reconstruct and understand the universe, from the mind of the people who form it.

In applied fields like health, integration of these different perspectives has been more and more frequent, due to the complexity and level of knowledge regarding human and social problems. Thus, the use of mixed or multiple methods is a tendency in nursing ${ }^{(1)}$.

\footnotetext{
${ }^{1}$ Member of the Editorial Board of the Latin American Journal of Nursing, Full Professor of the University of São Paulo at Ribeirão Preto College of Nursing, WHO Collaborating Centre for Nursing Research Development, Brazil, e-mail: furegato@eerp.usp.br.
} 
The growing interest of nursing in the search for knowledge, investments in research training and nursing knowledge production is shown by specialized journals, which publish more and better works in this area.

However, in research centers, support agencies and communication means, interest in quantitative research results still prevails. International publications are funded and supported by agencies that expect results and, hence, preferentially publish replicable and relevant studies.

The main criticism against qualitative methods refers to the lack of rigor as well as insufficient theoretical reflection. There are many studies that do not explain their methods clearly, nor the validity or reliability criteria of their search instruments, and write more about the researcher than the objects or subjects of the study, leaving the results at an intuitive level.

The choice of the theme of study, delimitation of the universe, variables of interest, subject selection criteria, techniques of data observation, collection, organization and treatment can be based on a qualitative search, with a possible numerical representation of data, even when accuracy or generalization are not targeted.

It is possible to measure phenomena and predict conducts using the qualitative approach. More than checking for hypotheses or theories, the goal of the research process is to identify them, like in research about knowledge, attitudes and opinions regarding a specific objective.

Psychometric tests, different kinds of scales, projective techniques and semantic analysis software are examples of resources researchers use to look for the sense and meaning of phenomena, through the subjects' expressions, measured with statistical resources.

It is supposed, in these cases, that many measurement and observation instruments are being developed, permitting conscious circulation among different research questions.

Integration of qualitative and quantitative methods, both in theory and applied research, values rigor in all scientific procedures, from the design to the conclusions.

Thus, the health research process uses techniques for data collection that evoke contextualized processes of daily life, and that permit progress in the understanding of the phenomena that determine the state of health and illness, as well as the therapeutic progress and care for these subjects.

Therefore, from the dichotomy positivism is based on towards the paradigm of scientific work that contemplates its whole complexity and the processual nature of reality, the focus should not be on the method, but on the design and on the seriousness of conducting the research or study.

On the one hand, qualitative information is supported by the relational context for understanding the subjectivity but, on the other hand, quantification assures the incorporation of decision techniques and procedures, increasing the range and value of explanations.

Accepting that knowledge is built through the relation of men with the world, the subject-object-subject formula is accepted, overcoming the dichotomy between pure object and pure subject, since the entire human reality implies multiple perspectives.

Overcoming this dualism enables the researcher to produce new levels of theoretical elaboration and contributions for the improvement of nursing knowledge and practices.

\section{REFERENCES}

Driessnack M, Souza VD, Mendes IAC. Revisão dos desenhos de pesquisa relevantes para a enfermagem. Parte 3: Métodos mistos e múltiplos. Rev Latino-Am. Enfermagem 2007;15(5):1048-9. 